\title{
Magnetic resonance imaging shrinkage patterns following neoadjuvant chemotherapy for breast carcinomas with an emphasis on the radiopathological correlations
}

\author{
KAORI TOMIDA $^{1}$, MITSUAKI ISHIDA ${ }^{2}$, TOMOKO UMEDA ${ }^{1}$, SACHIKO SAKAI ${ }^{1}$, YUKI KAWAI ${ }^{1}$, \\ TSUYOSHI MORI ${ }^{1}$, YOSHIHIRO KUBOTA ${ }^{1}$, EIJI MEKATA ${ }^{1}$, SHIGEYUKI NAKA ${ }^{1}$, \\ HAJIME ABE $^{1}$, HIDETOSHI OKABE ${ }^{2}$ and TOHRU TANI ${ }^{1}$ \\ Departments of ${ }^{1}$ Surgery and, ${ }^{2}$ Clinical Laboratory Medicine and Division of Diagnostic Pathology, \\ Shiga University of Medical Science, Otsu, Shiga 520-2192, Japan
}

Received February 12, 2014; Accepted June 4, 2014

DOI: $10.3892 / \mathrm{mco} .2014 .333$

\begin{abstract}
Preoperative neoadjuvant chemotherapy (NAC) is considered to be the standard treatment for locally-advanced breast carcinomas. Obtaining precise information regarding the tumor extent and distribution by imaging modalities to assess the success of breast-conserving surgery following NAC is extremely important. Analysis of the detailed radiopathological correlation of magnetic resonance imaging (MRI) following NAC has not been reported previously. The MRI and histopathological shrinkage patterns of residual breast carcinomas in 27 consecutive cases were analyzed following NAC and classified into five categories: Types I and II (concentric shrinkage with and without surrounding lesions, respectively); type III (shrinkage with residual multinodular lesions); type IV (diffuse contrast enhancement in whole quadrant); and non-visualization. The present study clearly demonstrated that the most common MRI shrinkage pattern was type I (11 cases), followed by type II and non-visualization, and the most common histopathological shrinkage pattern was type II (11 cases), followed by type III (8 cases). The concordance rate between MRI and pathological patterns was $48 \%$ and the worst concordance MRI pattern was type I. MRI is considered to be a useful method for evaluation of the residual carcinoma following NAC. However, the concordance rate was low in the MRI pattern I cases and tiny foci of residual carcinoma were present in half of the non-visualization cases, as shown by MRI. Therefore, the tumor extent must be completely resected for patients who undergo NAC, and postoperative radiation may be important for preventing local recurrence of breast carcinoma.
\end{abstract}

Correspondence to: Dr Tomoko Umeda, Department of Surgery, Shiga University of Medical Science, Seta Tsukinowa-cho, Otsu, Shiga 520-2192, Japan

E-mail: tomoko@belle.shiga-med.ac.jp

Key words: magnetic resonance imaging, neoadjuvant chemotherapy, shrinkage pattern, breast cancer

\section{Introduction}

Preoperative neoadjuvant chemotherapy (NAC) is considered the standard treatment for locally-advanced breast carcinomas (1-6). Several large randomized clinical studies have shown that NAC is effective as an adjuvant chemotherapy and permits breast-conserving surgery (1-6). The current roles of NAC include control and downstaging of primary breast tumors, as well as reducing micrometastatic diseases. Therefore, it is extremely important to obtain precise information regarding the extent and distribution of residual breast carcinomas following NAC by imaging modalities to assess the success of breast-conserving surgery.

Findings of previous studies have demonstrated the usefulness of imaging modalities for determining the tumor distribution of residual breast carcinoma following NAC (7-12). A study by Tozaki et al (9) analyzed computed tomography (CT) findings of tumor distribution patterns of locally-advanced breast carcinomas prior and subsequent to NAC. The distribution patterns of the enhancing lesions of breast carcinomas prior to NAC were classified into five categories: Solitary, grouped, separated, mixed and replaced lesions; and the shrinkage patterns subsequent to NAC were classified into three categories: Concentric shrinkage with or without surrounding lesions and shrinkage with residual multinodular lesions (9). The study concluded that CT classification of tumor distribution prior to NAC and shrinkage patterns subsequent to NAC is important for the evaluation of the residual lesions undergoing breast-conserving surgery (9). In addition, Kim et al (12) recently reported magnetic resonance imaging (MRI) patterns of breast carcinomas prior and subsequent to $\mathrm{NAC}$, and analyzed the correlation with pathological response grading. The shrinkage patterns of breast carcinomas following NAC were classified into five categories: Type I (concentric shrinkage without any surrounding lesion); type II (concentric shrinkage with surrounding lesions); type III (shrinkage with residual multinodular lesions); type IV (diffuse contrast enhancement in whole quadrant); and non-visualization (12). The study concluded that the type I pattern was the most frequently observed in the pathological responder group and type IV was more frequently found in the non-responder group (12). 
However, thus far, the analysis of the detailed radiopathological correlation of MRI prior and subsequent to NAC for breast carcinomas has not been reported. In the present study, a detailed analysis of the radiopathological correlation of residual breast carcinomas following NAC is reported, and the usefulness of MRI subsequent to NAC for breast-conserving surgery is discussed.

\section{Materials and methods}

Case selection. The study included 27 consecutive cases of breast carcinomas. The subjects who underwent NAC at Department of Surgery, Shiga University of Medical Science (Otsu, Shiga, Japan). In 20 cases, MRI was performed prior and subsequent to NAC, and in the remaining seven cases, MRI was performed only following NAC and CT was performed prior to NAC.

The mean age of the patients was 48.4 years (range, $29-66$ years). All the patients in the study were female. Table I shows the clinical features of the 27 cases.

The NAC regimens used were: taxane in five, taxane + anthracycline in 18 and taxane + anthracycline + trastuzumab in four cases. Mastectomy was performed in 9 cases and breast-conserving surgery was performed in 18 cases.

MRItechnique.MRIwas performed using 1.5T-and3T-scanners (Signa HD; GE Healthcare, Milwaukee, WI, USA) with the use of a dedicated breast coil. Patients underwent imaging in the prone position with breasts immobilized. Contrast material was injected in all the patients $(0.1 \mathrm{mmol} / \mathrm{kg}$ gadopentetate dimeglumine). The contrast-enhancement pattern was analyzed in the early phase.

CT technique. CT was obtained on a 64- (Aquilion CX; Toshiba Medical Systems, Otawara, Japan) or a 320-multidetector CT scanner (Aquilion ONE; Toshiba Medical Systems). Contrast material was injected in all the patients $(100 \mathrm{ml}$ of non-ionic contrast material).

Interpretation of MRI or CT findings. Four breast surgeons performed a consensus review of the breast MRI or CT examinations in all the cases. The initial contrast-enhancement pattern of breast carcinoma by MRI or CT prior to NAC was classified into five categories as suggested by Tozaki et al (9), i.e., solitary; grouped (localized lesion with linear, spotty, or linear and spotty enhancement); separated (multiple foci of contrast enhancement); mixed (grouped lesion with multiple foci); and replaced lesions (diffuse contrast enhancement in whole quadrant) (Fig. 1A).

The shrinkage pattern of breast carcinoma by MRI following NAC was classified into five categories as suggested by Kim et al (12): Type I (concentric shrinkage without any surrounding lesion); type II (concentric shrinkage with surrounding lesions); type III (shrinkage with residual multinodular lesions); type IV (diffuse contrast enhancement in whole quadrant); and non-visualization (Fig. 1B).

Histopathological evaluation. The histopathological diagnosis of breast carcinomas prior and subsequent to NAC was independently performed by two diagnostic pathologists. The
Table I. Clinical characteristics of breast carcinomas.

\begin{tabular}{lc}
\hline Characteristics & No. of cases $(\%)$ \\
\hline Initial clinical stage & \\
I & $1(4)$ \\
IIA & $7(26)$ \\
IIB & $17(63)$ \\
IIIA & $2(7)$ \\
IIIB & $0(0)$ \\
IIIC & $0(0)$ \\
IV & $0(0)$ \\
Surgical method & \\
Radical mastectomy & $9(33)$ \\
Partial mastectomy & $18(67)$ \\
Neoadjuvant chemotherapy regimen & \\
Taxane & $5(18)$ \\
Taxane + anthracycline & $18(67)$ \\
Taxane + anthracycline + trastuzumab & $4(15)$ \\
\hline
\end{tabular}

histopathological tumor regression pattern was semi-quantitatively evaluated using the Miller-Payne grading system as follows (13): Grade 1, no change or particular alteration to individual malignant cells and no reduction in overall cellularity; grade 2 , minor loss of tumor cells $(\leq 30 \%)$ but overall cellularity remained high; grade 3, between an estimated 30-90\% reduction in tumor cells; grade 4, a marked disappearance of tumor cells such that only small clusters or widely dispersed individual cells remained with a $>90 \%$ loss of tumor cells; and grade 5, no malignant cells identified in sections from the tumor site and only vascular fibroelastic stroma that often contains macrophages remains. However, intraductal components may be present (13). Patients showing Miller-Payne grades 3, 4 or 5 were classified as responders and patients showing grades 1 or 2 were classified as non-responders.

The histopathological pattern of the residual carcinoma was also classified by the same method as the MRI shrinkage pattern as suggested by Kim et al (12) (Fig. 1B).

\section{Results}

MRI or CT findings prior to NAC. Table II shows the initial enhancement patterns of MRI or CT of 27 cases of breast carcinomas prior to NAC. The most common initial contrast-enhancement pattern was solitary (18 cases) (Fig. 2A). The second most common pattern was grouped (five cases) (Fig. 2B), followed by separated (three cases) (Fig. 2C) and replaced patterns (one case) (Fig. 2D). There was no case showing a mixed pattern in the present study.

Histopathological findings of the biopsy specimen prior to $N A C$. All the cases were diagnosed as invasive ductal carcinoma of no special type by the biopsy specimen. No special type of invasive breast carcinoma, such as invasive lobular or mucinous carcinomas, was included in the present study.

MRI findings following NAC. Table II summarizes the MRI findings of the shrinkage patterns of breast carcinomas 
A

Initial enhancement patterns

Solitary

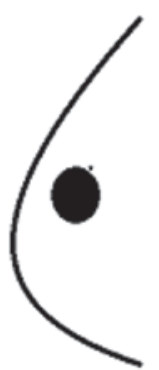

Grouped

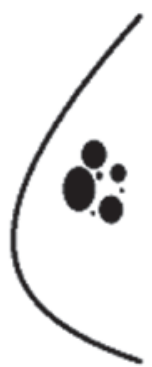

B Shrinkage patterns

Type I

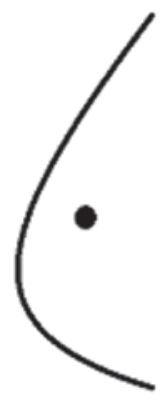

Type II

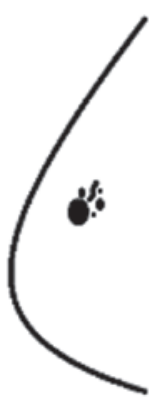

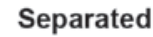

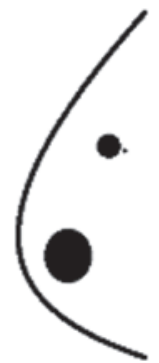

Type III

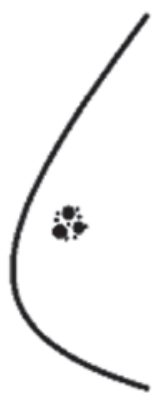

Replaced

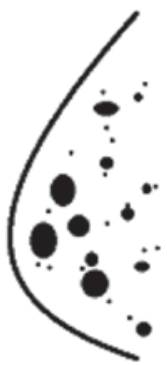

Type IV

Figure 1. Illustrated models of the initial enhancement and shrinkage patterns. (A) Initial enhancement patterns prior to neoadjuvant chemotherapy. Solitary, grouped, separated and replaced patterns. (B) Shrinkage patterns following neoadjuvant chemotherapy. Type I, concentric shrinkage without any surrounding lesion; type II, concentric shrinkage with surrounding lesions; type III, shrinkage with residual multinodular lesions; and type IV, diffuse contrast enhancement in whole quadrant.

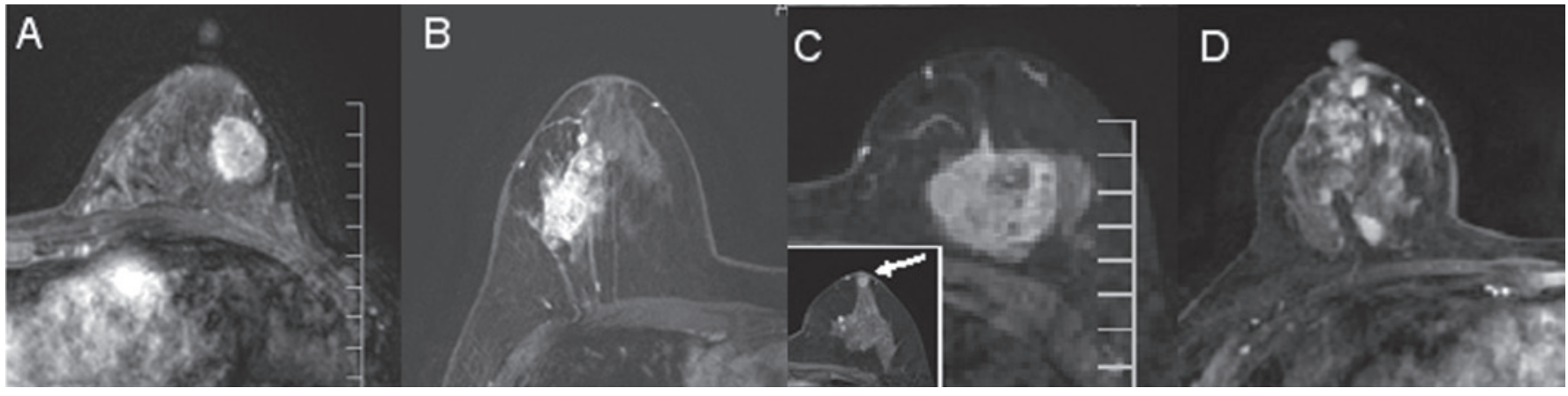

Figure 2. Magnetic resonance imaging of the initial enhancement patterns of breast carcinomas prior to neoadjuvant chemotherapy. (A) Solitary; (B) grouped; and (C) separated lesions. The arrow (inset) shows the daughter nodule. (D) Replaced lesion.

Table II. Correlation between initial contrast-enhancement patterns prior to neoadjuvant chemotherapy and shrinkage patterns.

Initial enhancement patterns

Shrinkage patterns Solitary Grouped Separated Replaced

\begin{tabular}{lllll}
\hline Non-visualization & 2 & 3 & 1 & 0 \\
Type I & 9 & 1 & 1 & 0 \\
Type II & 5 & 1 & 0 & 0 \\
Type III & 2 & 0 & 1 & 0 \\
Type IV & 0 & 0 & 0 & 1 \\
\hline
\end{tabular}

following NAC and the correlation between the initial contrast-enhancement patterns prior to NAC and the shrinkage patterns following NAC. The most common shrinkage pattern identified was type I (11/27 cases) (Fig. 3A). The second most common patterns identified were type II and non-visualization (6 cases each) (Fig. 3B and C). Three cases showed a type III pattern (Fig. 3D), while only one case showed type IV (Fig. 3E).

The most common shrinkage pattern of solitary lesions prior to NAC was type I (9/18 cases), in contrast to grouped lesions, which demonstrated that the non-visualization pattern (3/5 cases) was the most frequent. The replaced lesion showed the type IV shrinkage pattern. 
Histopathological findings following NAC. Table III summarizes the Miller-Payne grading system of the present study. The most common histopathological regression grade was grade 3 (10/27 cases). In four cases showing grade 5, no viable carcinoma cells and no residual intraductal component were observed in these cases. The rate of responder cases was $70.4 \%$ (19/27 cases) and that of non-responders was $29.6 \%$ (8/27 cases).

Table IV shows the radiopathological correlation of the shrinkage patterns of breast carcinomas following NAC in the study. The most common pathological shrinkage pattern of the residual carcinoma was type II (11/27 cases) (Fig. 4A), followed by type III ( 8 cases) (Fig. 4B). The concordance rate between the MRI pattern following NAC and pathological pattern was $48 \%$ : Non-visualization, three cases; type I, three (Fig. 4C); type II, four; type III, two; and type IV, one case (Fig. 4D). The worst concordance MRI pattern was type I as only three of the 11 cases corresponded to the pathological pattern, while four cases showed pathological type II and three cases showed pathological type III. In the residual carcinoma nodules, scar, accumulation of macrophages and hemosiderin deposition were observed in the cases showing MRI pattern type I and pathological pattern type II or III (Fig. 4E).

In the cases showing non-visualization by MRI following NAC, three of the six cases had no residual carcinoma by pathological examination and the remaining cases had only a few residual carcinomas by pathological examination, which showed pathological types II (two cases) and III (one case) (Fig. 4F).

Follow-up information subsequent to surgery. No local recurrence was observed in any of the 27 patients (median follow-up period, 32.9 months; range, 6-88 months).

\section{Discussion}

NAC is widely performed for patients with locally-advanced breast carcinomas in order to control and downstage the primary breast carcinomas. Therefore, accurate assessment regarding the extent and distribution of the residual breast carcinomas following NAC by imaging modalities is extremely important to assess the success of breast-conserving surgery. However, it has been reported that MRI and CT often over- or underestimate the extent and distribution of residual carcinomas following NAC (7-12).

A study by Tozaki et al (9) analyzed the usefulness of determining the breast carcinoma distribution prior to NAC and the shrinkage patterns following NAC by multidetector CT. The study evaluated 47 consecutive locally-advanced breast carcinomas from 46 patients and classified the distribution patterns of contrast enhancement prior to NAC by CT into 5 categories: Solitary; grouped; separated; mixed; and replaced lesions (9). Solitary lesions were observed in 15 cases, grouped in 12, separated in four, mixed in five and replaced in 11 cases in the series (9). The rates of grouped and replaced lesions in the present study were low compared to the series reported by Tozaki et al (9) [18.5\% were grouped lesions in the present study vs. $25.5 \%$ in the study by Tozaki et al (9) and $3.7 \%$ were replaced lesion in the present study vs. $23.4 \%$ in the study by Tozaki et al (9)]. However, the rates of the initial enhancement
Table III. Miller-Payne grading of the present study.

\begin{tabular}{lc} 
Grade & No. of cases \\
\hline 1 & 1 \\
2 & 7 \\
3 & 10 \\
4 & 5 \\
5 & 4 \\
\hline
\end{tabular}

patterns as reported by Kim et al (12), who also classified the initial enhancement patterns of MRI using the same methods suggested by Tozaki et al (9), fundamentally corresponded to the results of the present study. This difference may be due to a relatively high percentage of inflammatory carcinomas in the study reported by Tozaki et al (9).

Tozaki et al (9) also classified the shrinkage patterns with non-replaced lesions following NAC as concentric shrinkage with or without surrounding lesions, and shrinkage with residual multinodular lesions. The most common shrinkage pattern was concentric shrinkage without any surrounding lesions (16/28 cases showing complete or partial response by $\mathrm{CT}$ ), followed by shrinkage with residual multinodular lesions (five cases), complete response (four cases) and concentric shrinkage with surrounding lesions (three cases) (9). Furthermore, the study also reported that the assessment of the tumor extent following NAC by CT and histopathological examination of the residual carcinoma revealed that a deviation of $<2 \mathrm{~cm}$ in diameter was found in $88 \%$ of cases $(14 / 16)$, showing a pattern of concentric shrinkage without any surrounding lesions; $100 \%$ of cases (3/3), who had a pattern of concentric shrinkage with surrounding lesions; and none of the cases $(0 / 5)$ demonstrating a pattern of shrinkage with residual multinodular lesions (9). The cases showing a difference of $>2 \mathrm{~cm}$ in length from the histopathological examination were all underestimated, while no cases of overestimation were present in the study. Therefore, Tozaki et al (9) concluded that CT classification of shrinkage patterns following NAC is useful for evaluation of the residual lesions for undergoing breast-conserving surgery, particularly in the cases showing concentric shrinkage with or without surrounding lesions (9). However, CT underestimated the residual tumor extent by $>2 \mathrm{~cm}$ in the cases showing shrinkage with residual multinodular lesions (9). Therefore, a residual carcinoma in the surgical margins from the breast-conserving surgery is an extremely critical issue in these cases.

Recently, Kim et al (12) analyzed the MRI patterns of 56 consecutive breast carcinomas from 55 patients prior and subsequent to NAC and evaluated the correlation with pathological response grading. The shrinkage patterns of breast carcinomas following NAC were classified into five categories: Type I (concentric shrinkage without any surrounding lesion); type II (concentric shrinkage with surrounding lesions); type III (shrinkage with residual multinodular lesions); type IV (diffuse contrast enhancement in whole quadrant); and non-visualization (12). In that study, the most common shrinkage pattern was type I (29/56 cases), followed by type II (13 cases), type III (5 cases), type IV (4 cases) and non-visu- 
Table IV. Correlation between magnetic resonance imaging (MRI) shrinkage patterns and pathological patterns following neoadjuvant chemotherapy.

\begin{tabular}{lccccc}
\hline & \multicolumn{3}{c}{ Pathological patterns } \\
\cline { 2 - 5 } MRI patterns & Nonvisualization & Type I & Type II & Type III & Type IV \\
\hline Non-visualization & 3 & 0 & 2 & 1 & 0 \\
Type I & 1 & 3 & 4 & 3 & 0 \\
Type II & 0 & 0 & 4 & 2 & 0 \\
Type III & 0 & 0 & 1 & 0 & 0 \\
Type IV & 0 & 0 & 0 & 2 & 1 \\
\hline
\end{tabular}
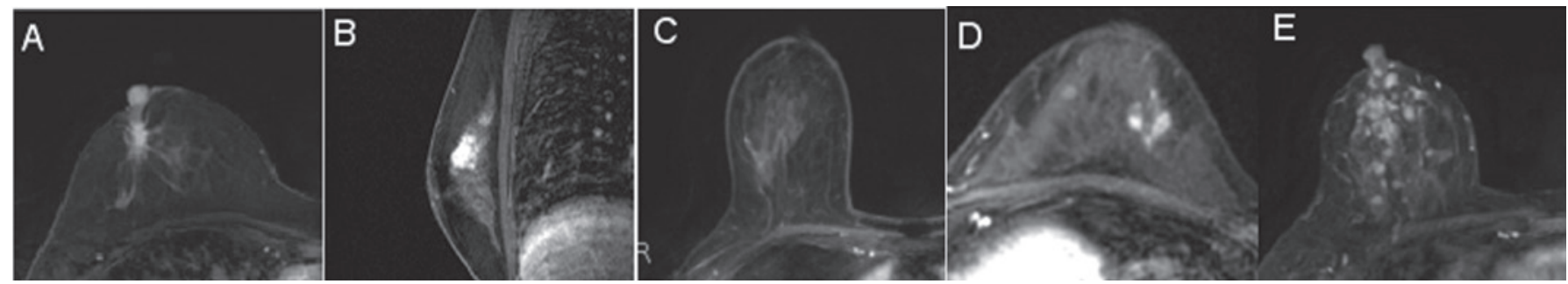

Figure 3. Magnetic resonance imaging of the shrinkage patterns of breast carcinomas following neoadjuvant chemotherapy. (A) Type I showing concentric shrinkage without any surrounding lesion. (B) Type II showing concentric shrinkage with surrounding lesions. (C) Non-visualization. (D) Type III showing shrinkage with residual multinodular lesions. (E) Type IV showing diffuse contrast enhancement in the whole quadrant.
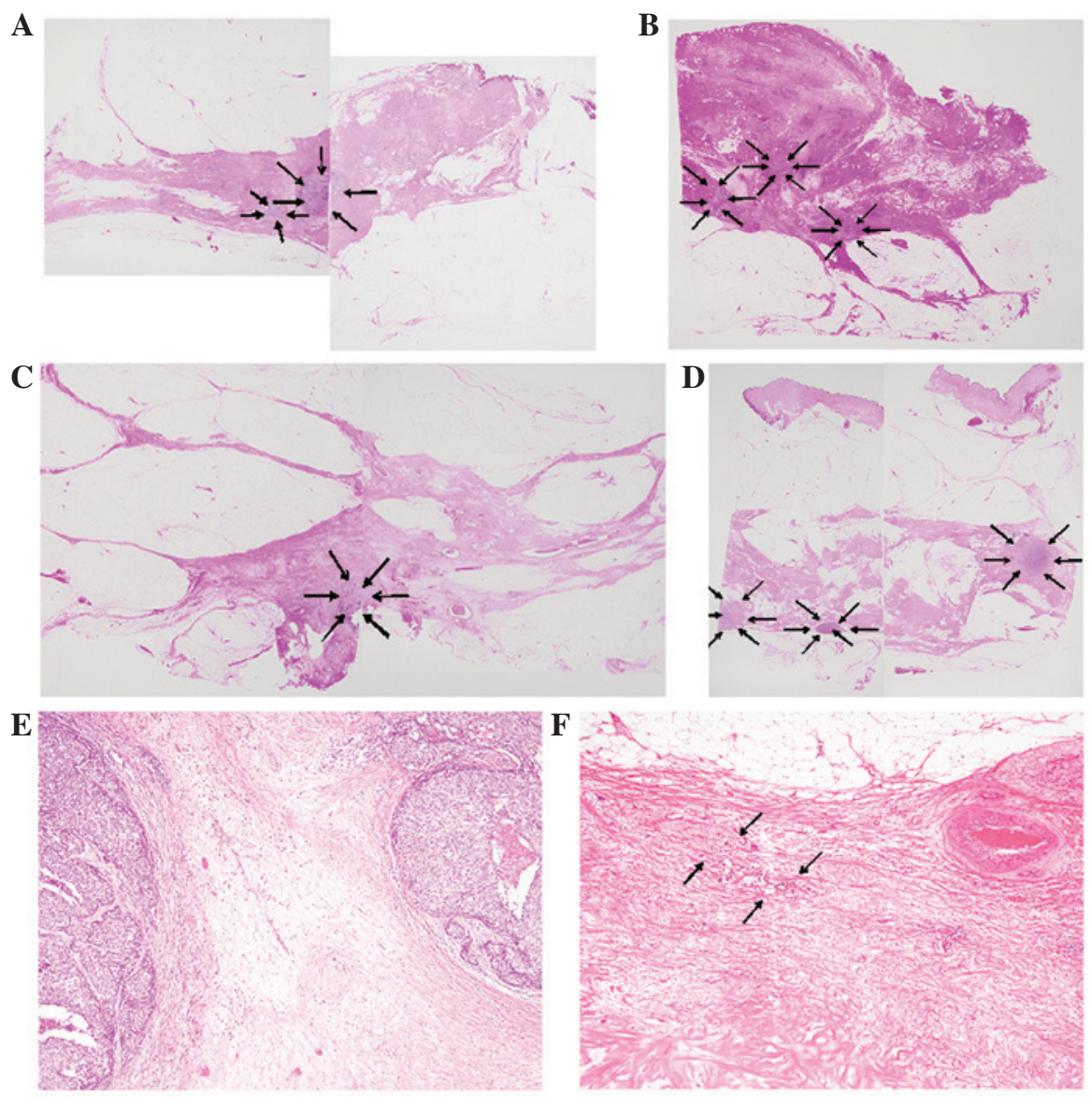

Figure 4. Panoramic view of the histopathological shrinkage patterns and features following neoadjuvant chemotherapy. (A) Pathological type II showing concentric shrinkage with surrounding lesions. (B) Pathological type III demonstrating shrinkage with residual multinodular lesions. (C) Pathological type I showing concentric shrinkage without any surrounding lesion. (D) Pathological type IV demonstrating multiple residual carcinomas (arrows, residual carcinoma cells); hematoxylin and eosin staining. (E) Scar, accumulation of macrophages and hemosiderin deposition are observed in the residual carcinoma nodules; hematoxylin and eosin staining (magnification, x40). (F) Only a few residual degenerative carcinoma cells are present within the scar and accumulation of macrophages; hematoxylin and eosin staining (magnification, $\mathrm{x} 40$ ). 
alization (3 cases) (12). The study concluded that type I was more frequently observed in the pathological responder group and type IV was more frequently noted in the non-responder group (12). Although the ratio of non-visualization was relatively high in the present study $(22 \%)$, the shrinkage patterns fundamentally corresponded to the results in the study by Kim et al (12).

To the best of our knowledge, the present study reports the first analysis regarding the radiopathological correlation between MRI shrinkage patterns subsequent to NAC and pathological residual tumor patterns. The histopathological patterns of the residual carcinoma following NAC were classified by the same method as the MRI shrinkage patterns suggested by Kim et al (12), as shown in Fig. 1B (13). The notable findings of the present study were: i) The most common histopathological shrinkage pattern was type II (11/27 cases), followed by type III (eight cases); ii) the concordance rate between MRI patterns following NAC and pathological patterns was $48 \%$; and iii) the worst concordance MRI pattern was type I ( $3 / 11$ cases).

Of the eight cases showing type I MRI and non-type I pathological patterns, four cases showed pathological type II and three cases showed pathological type III. This indicates that the residual tumor following NAC appeared as a concentric tumorous lesion without any surrounding lesions by MRI. However, histopathologically it did not form a tumorous lesion, but it was surrounded by a few or multinodular residual lesions and harboured fibrous scar with hemosiderin deposition and the accumulation of macrophages among the multinodular residual carcinoma nests, as shown in Fig. 4E. This may reflect a multifocal tumor shrinkage but not a concentric shrinkage pattern by NAC. In addition, of the six cases showing non-visualization by MRI following NAC, three cases had no residual carcinoma by pathological examination and the remaining cases had residual carcinoma, which were pathological types II (two cases) and III (one case). This may be due to the small amounts of residual carcinoma, as shown in Fig. 4F, which could not be detected by MRI.

In conclusion, the radiopathological correlation of breast carcinomas was analyzed prior and subsequent to NAC. MRI is considered to be a useful method for evaluating residual carcinomas following NAC (the concordance rate was 48\%). However, in the cases with MRI pattern I, the concordance rate was low and multinodular residual carcinomas were frequently present. Additionally, tiny foci of residual carcinoma were present in half of the non-visualization cases by MRI. Therefore, the tumor extent prior to NAC must be completely resected for patients who undergo NAC, particularly those with MRI patterns of type I, and non-visualization and postoperative radiation therapy may be important for preventing local recurrence of breast carcinomas.

\section{References}

1. Fisher B, Brown A, Mamounas E, et al: Effect of preoperative chemotherapy on local-regional disease in women with operable breast cancer: findings from National Surgical Adjuvant Breast and Bowel Project B-18. J Clin Oncol 15: 2483-2493, 1997.

2. Fisher B, Bryant J, Wolmark N, et al: Effect of preoperative chemotherapy on the outcome of women with operable breast cancer. J Clin Oncol 16: 2672-2685, 1998.

3. Mamounas EP and Fisher B: Preoperative (neoadjuvant) chemotherapy in patients with breast cancer. Semin Oncol 28: 389-399, 2001.

4. Goldhirsch A, Glick JH, Gelber RD, Coates AS and Senn HJ: Meeting highlights: International Consensus Panel on the Treatment of Primary Breast Cancer. Seventh International Conference on Adjuvant Therapy of Primary Breast Cancer. J Clin Oncol 19: 3817-3827, 2001.

5. Mauri D, Pavlidis N and Ioannidis JP: Neoadjuvant versus adjuvant systemic treatment in breast cancer: a meta-analysis. J Natl Cancer Inst 97: 188-194, 2005.

6. Mieog JS, van der Hage JA and van de Velde CJ: Preoperative chemotherapy for women with operable breast cancer. Cochrane Database Syst Rev 2: CD005002, 2007.

7. Rieber A, Zeitler H, Rosenthal H, et al: MRI of breast cancer: influence of chemotherapy on sensitivity. Br J Radiol 70: 452-458, 1997.

8. Wasser K, Sinn HP, Fink C, et al: Accuracy of tumor size measurement in breast cancer using MRI is influenced by histological regression induced by neoadjuvant chemotherapy. Eur Radiol 13: 1213-1223, 2003.

9. Tozaki M, Kobayashi T, Uno S, et al: Breast-conserving surgery after chemotherapy: value of MDCT for determining tumor distribution and shrinkage pattern. AJR Am J Roentogenol 186: 431-439, 2006.

10. Bahri S, Chen JH, Mehta RS, et al: Residual breast cancer diagnosed by MRI in patients receiving neoadjuvant chemotherapy with and without bevacizumab. Ann Surg Oncol 16: 1619-1628, 2009

11. Woodhams R, Kakita S, Hata $\mathrm{H}$, et al: Identification of residual breast carcinoma following neoadjuvant chemotherapy: diffusion-weighted imaging - comparison with contrast-enhanced MR imaging and pathological findings. Radiology 254: 357-366, 2010.

12. Kim TH, Kang DK, Yim H, Jung YS, Kim KS and Kang SY: Magnetic resonance imaging patterns of tumor regression after neoadjuvant chemotherapy in breast cancer patients: correlation with pathological response grading system based on tumor cellularity. J Comput Assist Tomogr 36: 200-206, 2012.

13. Ogston KN, Miller ID, Payne S, et al: A new histological grading system to assess response of breast cancers to primary chemotherapy: prognostic significance and survival. Breast 12: 320-327, 2003. 